\title{
Fast algorithm for generating sorted contour strings
}

Norman L. Jones

Brigham Young University - Provo, njones@byu.edu

Michael J. Kennard

Brigham Young University - Provo

Alan K. Zundel

Brigham Young University - Provo

Follow this and additional works at: https://scholarsarchive.byu.edu/facpub

Part of the Geotechnical Engineering Commons

\section{Original Publication Citation}

Jones, Norman L., Michael J. Kennard, Alan K. Zundel, "Fast algorithm for generating sorted contour strings,"Computers and Geosciences, Vol. 26, pp. 831-837, 2000.

\section{BYU ScholarsArchive Citation}

Jones, Norman L.; Kennard, Michael J.; and Zundel, Alan K., "Fast algorithm for generating sorted contour strings" (2000). Faculty Publications. 4298.

https://scholarsarchive.byu.edu/facpub/4298

This Peer-Reviewed Article is brought to you for free and open access by BYU ScholarsArchive. It has been accepted for inclusion in Faculty Publications by an authorized administrator of BYU ScholarsArchive. For more information, please contact ellen_amatangelo@byu.edu. 


\title{
Fast algorithm for generating sorted contour strings
}

\author{
Norman L. Jones*, Michael J. Kennard, Alan K. Zundel \\ Environmental Modeling Research Laboratory, Brigham Young University, 242 CB BYU, Provo, Utah, 84602, USA
}

Received 28 June 1999; received in revised form 1 February 2000; accepted 1 February 2000

\begin{abstract}
Automatic generation of contours for graphical display and map plotting has been studied extensively since the early days of computing. The individual segments making up a contour line are often determined by subdividing the object of interest into small triangles and computing the contours assuming a linear variation on each triangle. However, efficient storage of contour data and the need to place labels (automatically) or to smooth the contours require that the contours be generated in continuous strings of segments. A simple approach to generate such strings is to sort the randomly generated contour segments. Since sorting can be time-consuming, the majority of previous approaches are contour-tracing algorithms that traverse the surface and generate the contour in a continuous sequence of segments. In this paper, we present a new sorting algorithm. The algorithm is relatively easy to implement, can be applied to any type of surface, and works for both $2 \mathrm{D}$ and $3 \mathrm{D}$ objects. The algorithm is significantly faster than the contour tracing approach, particularly when large numbers of segments are involved. (C) 2000 Elsevier Science Ltd. All rights reserved.
\end{abstract}

Keywords: Contour generation; Contour sorting algorithm

\section{Introduction}

One of the most frequent programming applications in the geosciences is the automated generation of contour plots. Data to be contoured can reside on a wide variety of object types including digital elevation models, triangulated irregular networks (TINs), twoand three-dimensional finite-difference grids, and finiteelement meshes. Regardless of the object type, the simplest approach to generating contours is to use a divide and conquer approach. The surface of the object is subdivided into a set of triangles, each triangle having a data value at each of its three vertices. Some objects

\footnotetext{
* Corresponding author. Tel.: +1-801-378-2812; fax: +1801-378-2478.

E-mail address: njones@et.byu.edu (N.L. Jones).
}

are naturally composed of triangles (TINs and triangular finite-element meshes) and some must be subdivided. For example, for a two-dimensional grid, each grid cell can be subdivided into two triangles (Fig. 1a) or a data value can be interpolated to the cell center and the grid cell can be subdivided into four triangles (Fig. 1b). For superior results, a data value can also be interpolated to the midpoint of the cell edges and the cell can be subdivided into eight triangles (Fig. 1c). At this point, a set of linear contour segments (one per contour value) can easily be generated from each triangle. A simple algorithm can be used to linearly interpolate along the three edges of the triangle to find the intersection of the contour values with the triangle edges and connect the intersections to construct the linear segments (Watson, 1982). This process is repeated for all triangles on the surface and the con-

0098-3004/00/\$ - see front matter (C) 2000 Elsevier Science Ltd. All rights reserved.

PII: S0098-3004(00)00009-1 
tour plot is generated by displaying the complete set of linear segments.

The divide and conquer approach results in a randomly ordered set of linear contour segments. For simple applications, this is sufficient. However, in many instances, it is advantageous to have the contour segments organized into strings or loops representing the full length of the contour line. Contour strings are necessary for two popular contour plotting options: labels and splines. Plotting contour labels involves placing a set of short text strings corresponding to the contour value on the contour string. For best results, the contour labels should not be too crowded or too sparse and they must be evenly spaced. To perform the calculations required to space the labels correctly, the contours must be organized into strings. The other application is spline contouring. Spline contouring is a simple mathematical technique for "smoothing" the contours (Lancaster and Salkauskas, 1986). To compute the spline functions, a gradient (slope in the $x$ and $y$ direction) must be computed for each vertex (endpoint of a segment) in the contour string. This also requires that the segments be organized in a sequential fashion along the contour string.

Traditionally, two approaches have been used to generate contours in strings (Watson, 1992). One approach is to generate a list of contour segments for a given contour interval and then use a sorting algorithm to organize the contour segments into strings. The simplest sorting algorithm involves selecting a starting segment and then searching the remaining contour segments in the list to find one with a vertex whose coordinates match one of the vertices on the starting segment. This is repeated until all of the segments are sorted. The drawback of this sorting approach is that the algorithm is $\mathrm{O}\left(\mathrm{N}^{2}\right)$ where $\mathrm{N}$ is the number of contour segments. To improve the efficiency, most programmers have elected to use a different approach to generate the contours. Rather than generate a random set of segments and then order the segments, the contours are initially generated in a sequential fashion. This is accomplished by finding a location on the object where a contour string begins and then tracing the segment from triangle to triangle until the end of the string is reached or until the loop closes on itself. When the contour string intersects a

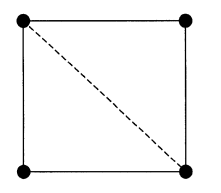

(a)

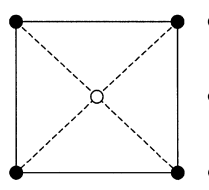

(b)

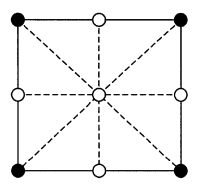

(c)
Fig. 1. Cell subdivision (a) two subtriangles, (b) four subtriangles, (c) eight subtriangles. triangle at a node, additional logic must be used to determine which adjacent triangle the contour line goes through. In addition to the segment-tracing logic, the algorithm must include an efficient method for determining where to begin the contour tracing and determining when all available strings have been located and traced. If the initial object needs to be subdivided into triangles, the tracing approach requires that all computed triangles are stored in memory, along with information describing the adjacency relationships among the triangles. With the sorting approach, the triangles and the adjacency relationships do not need to be stored in memory (the adjacency information does not need to be computed at all). Thus, while this approach is faster than the brute force sorting approach, it is more complicated, memory intensive, and costly to implement. Examples of the contour tracing approach can be found in Batcha and Reese (1964), Buys et al. (1991), Kok and Begin (1981), Lodwick and Whittle (1970), Sabin (1980), and Yates (1987).

Whereas most attempts to improve contouring efficiency have focused on contour tracing algorithms, another approach is to develop more efficient contour sorting algorithms. Anderson (1983) and Nickerson et al. (1999) describe fast quadtree sorting methods that could be adapted specifically to contouring, but these approaches work only in two dimensions. The algorithm we describe works equally well for two and three dimensions, and is $\mathrm{O}(\mathrm{N} \log \mathrm{N})$. The algorithm requires additional memory (compared to a brute force sort) but the storage requirements are not substantial. The algorithm is simpler to implement than the contour tracing approach and is significantly faster than both contour tracing and the brute force methods. The algorithm has been implemented in C.

\section{Improved sorting algorithm}

The basic steps involved in the new sorting algorithm are as follows:

1. Traverse the triangles, creating and storing the contour segments in a linked list.

2. Store the vertices of the contour segments in an array.

3. Sort the array of vertices using an efficient sorting algorithm. Sort first by $x$ and then by $y$. For 3D cases, the array is also sorted by $z$.

4. Transform the array into an embedded, doublylinked list.

5. Parse the list of vertices to remove all duplicate vertices.

6. Use a special technique to parse through the vertices and link them into open or closed contour strings. 
A flow chart of the algorithm is shown in Fig. 2. We will now explain each of these steps in more detail.

\subsection{Creating the contour segments}

Before the contour segments can be sorted, they are first generated and stored in a linked list. Each triangle in the surface is traversed and the linear contour segments from each triangle are added to the list. The segments are stored in the list in the order that they are generated.
Table 1

Information stored in vertex record

\begin{tabular}{ll}
\hline Item & Description \\
\hline$x$ & $x$ coordinate of vertex \\
$y$ & $y$ coordinate of vertex \\
$z$ & $z$ coordinate of vertex \\
s1 & First segment attached to vertex \\
s2 & Second segment attached to vertex \\
prev & Pointer to previous vertex in linked list \\
next & Pointer to next vertex in linked list \\
\hline
\end{tabular}

\subsection{Building the vertex array}

The basic idea behind the sorting algorithm is to generate an alternate representation of the contour segments that can be stored in a supplemental array. A highly efficient algorithm can then be used to sort the array. This is accomplished by focusing attention on the vertices of the segments, rather than on the segments themselves. The array consists of one structure for each vertex. Two vertices are stored in the array for each contour segment (even though most of the vertices are shared). The vertex structure contains the information shown in Table 1. The first three items are the coordinates of the vertex. The next two items are pointers to the two segments attached to the vertex. The final two items are used to build an embedded linked list after the initial array sort is complete.

When the array is first constructed, the first segment pointer, s1, is set to point to the segment attached to the vertex. This is the segment in the original list of segments generated by traversing the triangles. The other segment pointer, s2, is set to NULL. The previous and next pointers are ignored for now.

To illustrate the data structure and the sorting process, we will use the sample contour strings shown in Fig. 3. The example includes both an open loop and a closed loop. For simplicity, each vertex is identified by a letter rather than the vertex coordinates. The segments are identified by numbers.

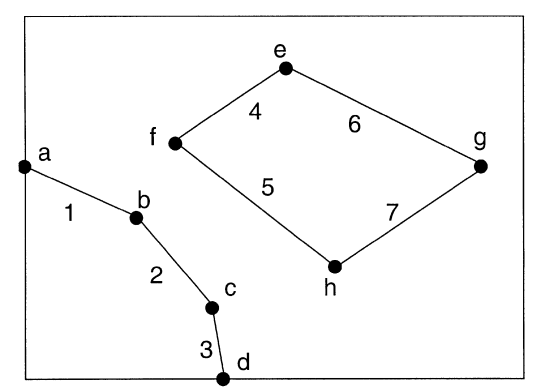

Fig. 3. Sample set of contour segments.

Fig. 2. Flow chart of sorting algorithm. 
In addition to the array of vertices, the sorting algorithm uses a linked list of contour segments. Both of the data structures are shown in Table 2 just after the data structures are built. The initial ordering of the segments $(4,3,2,5,7,6,1)$ corresponds to the order in which the segments were initially generated. The segment list is a temporary holding bin for the original unsorted contour segments. Each contour segment contains the $x y z$ coordinates of the two vertices defining the segment. As the sorting algorithm proceeds, the segments in this list are organized into two sorted loops, one for each of the two contour strings shown in Fig. 3. At this point the vertex array contains the vertices in random order. Note that each of the endpoints in the closed loop and the interior vertices in the closed loop are listed twice, while the endpoints of the open loop (a and d) are listed once.

\subsection{Sorting the vertices}

Once the array is constructed, the next step is to sort the array. The array is sorted first by $x$ and then by $y$. If the contour segments are generated from a 3D object, the array is also sorted by $z$. The purpose of this sort is not to directly build the contour strings. Rather, once the vertices are sorted by $x, y$, and $z$, a highly efficient algorithm can be used to build the contour strings. The array sort is accomplished using the standard qsort algorithm (Kelley and Pohl, 1984) provided in $\mathrm{C}$. The qsort algorithm is $\mathrm{O}(\mathrm{N} \log \mathrm{N})$. The comparison function passed to the qsort function first compares $x$ values. If the $x$ values are equal, it compares $y$ values. If the $y$ values are equal, it compares $z$ values. The data structures after the sorting process are shown in Table 3.

\subsection{Building the embedded linked list}

Once the vertex array is sorted on $x, y$, and $z$, the next step is to build an embedded linked list inside the vertex array. The linked list provides fast traversal and editing of the items in the list as the contour strings

Table 2

Initial state of data structures

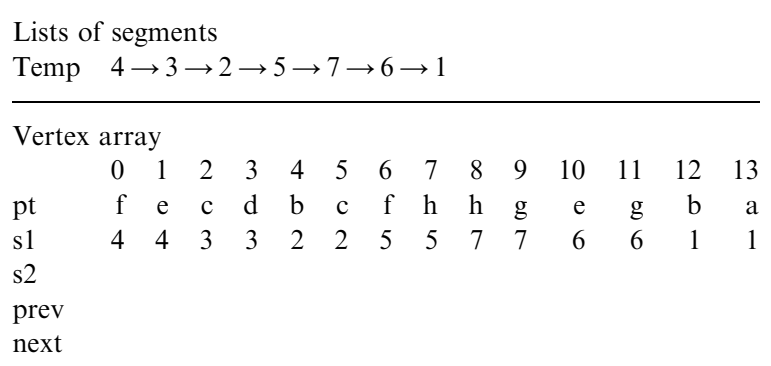

Table 3

Vertex array after sorting on $x$ and $y$

Lists of segments
Temp $\quad 4 \rightarrow 3 \rightarrow 2 \rightarrow 5 \rightarrow 7 \rightarrow 6 \rightarrow 1$

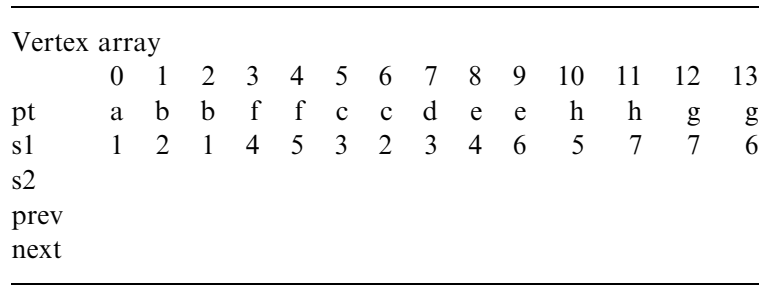

are being constructed. In order to simplify the process of deleting items from the list, the list is doubly linked (each item contains a pointer to both the next and the previous item in the list). The list is initially established simply by traversing the array from the beginning and initializing the previous and next pointers. The vertex array after the linked list is initially constructed is shown in Table 4.

\subsection{Removing duplicate vertices}

Before building the contour strings, there is one more adjustment to be made to the list. All vertices in closed loops and interior vertices in open loops are included twice in the list. The next step is to remove duplicate vertices and initialize the second segment pointer (s2). Because of the $x$ and $y$ sorting process, all duplicate vertices will be adjacent to one another in the array. We start at the beginning of the list and traverse the vertices. For each vertex, if the $x y$ coordinates of the current vertex are identical (within a small distance tolerance) to the coordinates of the next vertex in the array, the s2 pointer of the current vertex is set to point to the segment pointed to by the $\mathrm{s} 1$ pointer of the second vertex. The previous and next pointers are adjusted so that the second vertex is removed from the embedded linked list (it is still in the array but it is not linked). If the next vertex is not the same, the ver-

Table 4

Vertex array after initialization of embedded linked list

Lists of segments

Temp $\quad 4 \rightarrow 3 \rightarrow 2 \rightarrow 5 \rightarrow 7 \rightarrow 6 \rightarrow 1$

\begin{tabular}{|c|c|c|c|c|c|c|c|c|c|c|c|c|c|c|}
\hline \multicolumn{15}{|c|}{ Vertex array } \\
\hline & 0 & 1 & 2 & 3 & 4 & 5 & 6 & 7 & 8 & 9 & 10 & 11 & 12 & 13 \\
\hline $\mathrm{pt}$ & $\mathrm{a}$ & $\mathrm{b}$ & $\mathrm{b}$ & f & $\mathrm{f}$ & $\mathrm{c}$ & $\mathrm{c}$ & $\mathrm{d}$ & $\mathrm{e}$ & $\mathrm{e}$ & $\mathrm{h}$ & $\mathrm{h}$ & $\mathrm{g}$ & $g$ \\
\hline s1 & 1 & 2 & 1 & 4 & 5 & 3 & 2 & 3 & 4 & 6 & 5 & 7 & 7 & 6 \\
\hline \multicolumn{15}{|l|}{$\mathrm{s} 2$} \\
\hline prev & null & 1 & 2 & 3 & 4 & 5 & 6 & 7 & 8 & 9 & 10 & 11 & 12 & \\
\hline next & 1 & 2 & 3 & 4 & 5 & 6 & 7 & 8 & 9 & 10 & 11 & 12 & 13 & nul \\
\hline
\end{tabular}


Table 5

Vertex array after removal of duplicate vertices

Lists of segments

Temp $\quad 4 \rightarrow 3 \rightarrow 2 \rightarrow 5 \rightarrow 7 \rightarrow 6 \rightarrow 1$

Vertex array

\begin{tabular}{lrrrrrrrrrrrrrr} 
& 0 & 1 & 2 & 3 & 4 & 5 & 6 & 7 & 8 & 9 & 10 & 11 & 12 & 13 \\
pt & $\mathrm{a}$ & $\mathrm{b}$ & $\mathrm{b}$ & $\mathrm{f}$ & $\mathrm{f}$ & $\mathrm{c}$ & $\mathrm{c}$ & $\mathrm{d}$ & $\mathrm{e}$ & $\mathrm{e}$ & $\mathrm{h}$ & $\mathrm{h}$ & $\mathrm{g}$ & $\mathrm{g}$ \\
$\mathrm{s} 1$ & 1 & 2 & 1 & 4 & 5 & 3 & 2 & 3 & 4 & 6 & 5 & 7 & 7 & 6 \\
$\mathrm{~s} 2$ & & 1 & & 5 & & 2 & & & 6 & & 7 & & 6 & \\
prev & null & 0 & - & 1 & - & 3 & - & 5 & 7 & - & 8 & - & 10 & - \\
next & 1 & 3 & - & 5 & - & 7 & - & 8 & 10 & - & 12 & - & null & - \\
\hline
\end{tabular}

tex is the endpoint of an unclosed loop and we leave the s2 pointer as NULL. The state of the vertex array after the duplicate point removal is shown in Table 5.

From this point forward, all traversals of the vertex list are made using the pointers in the embedded linked list. This speeds up the traversal and ensures that only vertices that have not been deleted from the list are considered.

\subsection{Linking vertices into strings}

At this point, we are ready to traverse the linked list in the vertex array and build contour strings. When building the loops, we must distinguish between the open loops and the closed loops since the loop building process varies for each type. We must first build the open loops. The first step is to find the endpoint of an open loop. Such an endpoint can be distinguished from other vertices since it has only one adjacent segment pointer. To find such an endpoint, we start at the beginning of the list and traverse the vertices until we find the first vertex where the s2 pointer is NULL (vertex "a" in our example).

Once we find the endpoint of an open loop we create a new contour segment list and move the first segment (the segment corresponding to the s1 pointer of the vertex) from the original list to the new list. We also remove the vertex from the embedded linked list by adjusting the previous and next pointers. We then use the s1 pointer and go to the segment (segment 1 in our example) and see if the opposite vertex is in the $+x$ or $-x$ direction. If the $x$ values are the same, we check the $y$ values to see which direction to proceed. We then go forward or backward in the vertex list (forward, in our case) using the linked list pointers until we find a segment where either the $\mathrm{s} 1$ or the s2 pointer corresponds to the current segment. In our example, the next vertex in the list, vertex $b$, has its $s 2$ pointer pointing to the current segment, segment 1 . We then look at the opposite segment pointer for this vertex ( $\mathrm{s} 1$ in our example) and move this segment (segment 2 in our example) from the temp list to the new list. Once
Table 6

Data structures after construction of open loop

\begin{tabular}{|c|c|c|c|c|c|c|c|c|c|c|c|c|c|c|c|}
\hline $\begin{array}{l}\text { Lists of } \\
\text { Temp } \\
\text { Loop } 1\end{array}$ & $\begin{array}{l}\text { segn } \\
4- \\
1-\end{array}$ & $\begin{array}{l}\text { nen } \\
\rightarrow 5 \\
\rightarrow 2\end{array}$ & $\stackrel{\text { ts }}{\rightarrow}$ & $\rightarrow 6$ & & & & & & & & & & & \\
\hline \multicolumn{16}{|c|}{ Vertex array } \\
\hline & 0 & 1 & 2 & 3 & 4 & 5 & 6 & & & 8 & 9 & 10 & 11 & 12 & 13 \\
\hline $\mathrm{pt}$ & $\mathrm{a}$ & b & $\mathrm{b}$ & $\mathrm{f}$ & f & c & c & 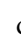 & & $\mathrm{e}$ & e & $\mathrm{h}$ & $\mathrm{h}$ & $\mathrm{g}$ & g \\
\hline s1 & 1 & 2 & 1 & 4 & 5 & 3 & 2 & & & 4 & 6 & 5 & 7 & 7 & 6 \\
\hline s2 & & 1 & & 5 & & 2 & & & & 6 & & 7 & & 6 & \\
\hline prev & - & - & - & null & - & - & - & & & 3 & - & 8 & - & 10 & - \\
\hline next & - & - & - & 8 & - & - & - & & & 10 & - & 12 & - & null & - \\
\hline
\end{tabular}

again, we also change the previous and next pointers in the vertex list to delete this vertex from the array. Then we look at the segment we just added (segment 2) and look at its opposite vertex (opposite from b is vertex c) and go forward or backward until we find that opposite vertex. This process will continue until we come to a vertex where the sl pointer matches the segment we are looking for and the s2 pointer is NULL. This is the end of the open loop. After processing the first open loop, our array and loop lists are as shown in Table 6.

After the first open loop is completed, the process is repeated for the next open loop. At some point, all open loops will be found and constructed. This state is detected when the entire vertex list is traversed and none of the remaining vertices have only one adjacent segment. Since our example has only one open loop, this is the state of the vertex list shown in Table 6 .

Once the open loops are completed, the next step is to construct the closed loops. The closed loops are built using the same process used to build the open loops, except that starting and ending the loop is handled differently. Closed loops can be started at any location. To start the loop, we start at the first vertex in the list and examine the two segments adjacent to

Table 7

Data structures after construction of closed loop

Lists of segments

Temp

Loop $1 \quad 1 \rightarrow 2 \rightarrow 3$

Loop $2 \quad 4 \rightarrow 6 \rightarrow 7 \rightarrow 5$

\begin{tabular}{|c|c|c|c|c|c|c|c|c|c|c|c|c|c|c|}
\hline \multicolumn{15}{|c|}{ Vertex array } \\
\hline & 0 & 1 & 2 & 3 & 4 & 5 & 6 & 7 & 8 & 9 & 10 & 11 & 12 & 13 \\
\hline $\mathrm{pt}$ & $\mathrm{a}$ & $\mathrm{b}$ & $\mathrm{b}$ & $\mathrm{f}$ & $\mathrm{f}$ & $\mathrm{c}$ & $\mathrm{c}$ & $\mathrm{d}$ & $\mathrm{e}$ & $\mathrm{e}$ & $\mathrm{h}$ & $\mathrm{h}$ & $\mathrm{g}$ & $\mathrm{g}$ \\
\hline s1 & 1 & 2 & 1 & 4 & 5 & 3 & 2 & 3 & 4 & 6 & 5 & 7 & 7 & 6 \\
\hline s1 & & 1 & & 5 & & 2 & & & 6 & & 7 & & 6 & \\
\hline prev & - & - & - & - & - & - & - & - & - & - & - & - & - & - \\
\hline next & - & - & - & - & - & - & - & - & - & - & - & - & - & - \\
\hline
\end{tabular}




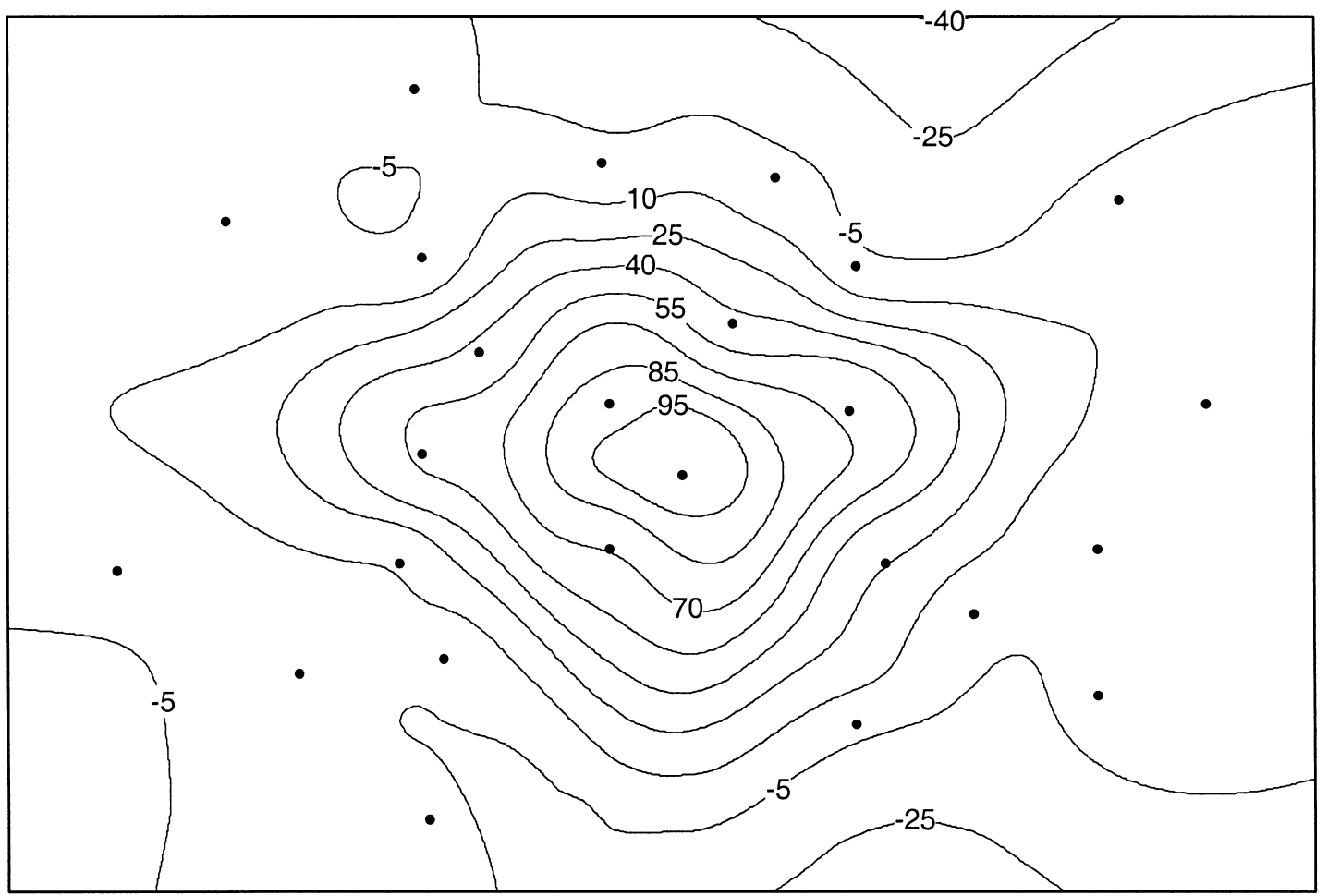

Fig. 4. Scatter points and 2D grid used in algorithm comparison.

the starting vertex using the s1 and s2 pointers. One segment is selected as the first segment in the list and one is marked as the ending segment. We then begin building the loop. As we build the loop, we check each new segment added to the list and stop when we reach the ending segment. This process is repeated until all closed loops are found and the vertex list is empty as shown in Table 7. The vertex array is then freed from

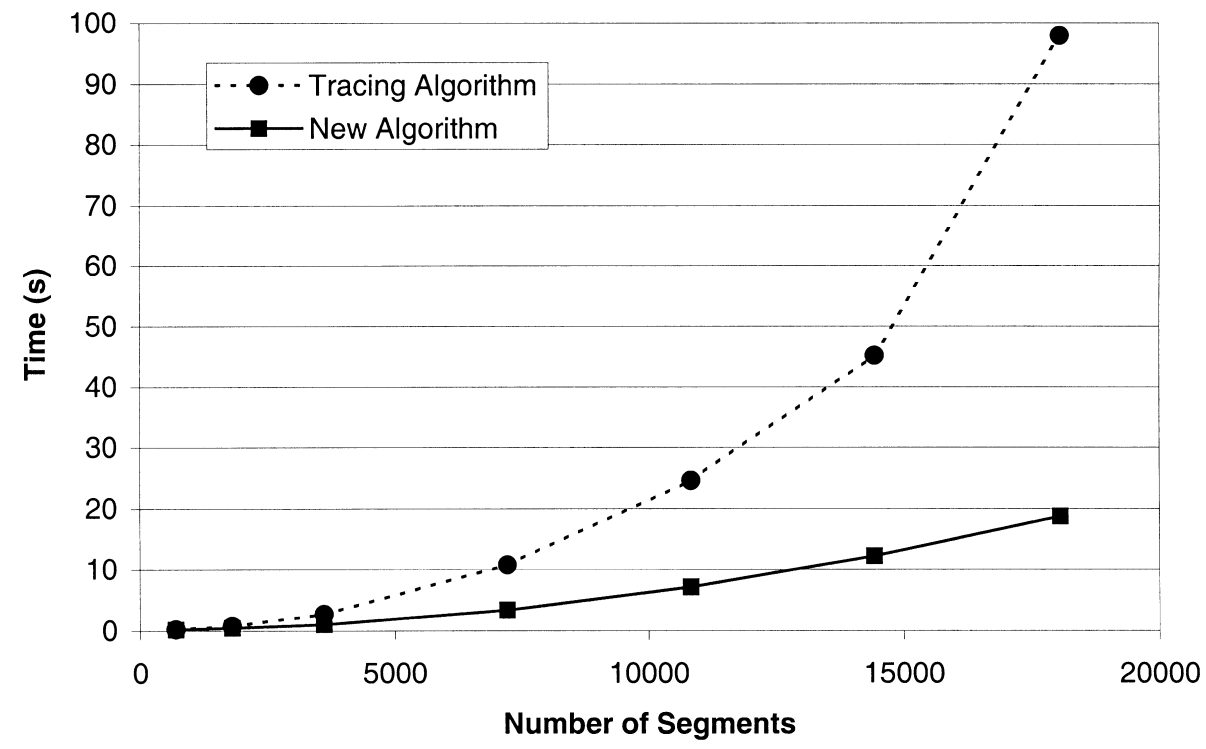

Fig. 5. Computation time vs number of contour segments for test case. 
memory, and the process is repeated on the set of segments corresponding to the next contour value.

\section{Algorithm performance}

To gauge the performance of the new algorithm, we conducted a test comparing the new sorting algorithm to a more traditional contour tracing algorithm. The data set used in the test was a set of $2 \mathrm{D}$ scatter points, each point having a measured elevation value. The points were interpolated to the nodes of a 2D finite-difference grid. The resulting contours are shown in Fig. 4.

For a given contour interval, the grid was contoured using both algorithms. The total number of segments in the contour strings was computed and the time required to generate the contour strings using each algorithm was recorded. We then increased the number of segments by altering the grid density. We repeated this process several times. The tests were conducted on a $400 \mathrm{MHz}$ Pentium II PC. The results of the algorithm comparison are shown in Fig. 5. The new algorithm is much faster than the tracing algorithm, particularly for situations with a large number of contour segments.

\section{Conclusions}

We have presented a new algorithm for sorting contour segments into contiguous strings. The algorithm is relatively easy to implement and is highly efficient. It requires a supplemental vertex array but the array is one-dimensional and the memory requirements are minimal. The algorithm can be applied to any type of geometric object and it can also be used for either 2D or $3 \mathrm{D}$ contours. We have used the algorithm to con- tour TINs, 2D grids, 2D meshes, and the exterior surfaces of 3D grids and meshes.

\section{References}

Anderson, D.P., 1983. Techniques for reducing pen plotting time. ACM Transactions on Graphics 2 (3), 197-212.

Batcha, J.P., Reese, J.R., 1964. Surface determination and automatic contouring for mineral exploration, extraction and processing. Colorado School of Mines Quarterly 14 (4), 187-194.

Buys, J., Messerschmidt, H.J., Botha, J.F., 1991. Including known discontinuities directly into a triangular irregular mesh for automatic contouring purposes. Computers \& Geosciences 17 (7), 875-881.

Kelley, A.L., Pohl, I., 1984. A Book On C: Programming in C, 2nd ed. Benjamin/Cummings Publishing Company, California 525 pp.

Kok, R., Begin, J., 1981. Evaluation of automatic contouring methods for drainage design. Transactions of the ASAE 24 (1), 87-96, 102.

Lancaster, P., Salkauskas, K., 1986. Curve and Surface Fitting: An Introduction. Academic Press, London, 280 pp.

Lodwick, G.D., Whittle, J., 1970. A technique for automatic contouring field survey data. The Australian Computer Journal 2 (3), 104-109.

Nickerson, B.G., Judd, P.A., Mayer, L.A., 1999. Data structures for fast searching of SEG-Y seismic data. Computers \& Geosciences 25 (2), 179-190.

Sabin, M.A., 1980. Contouring - a review of methods for scattered data. In: Brodlie, K.W. (Ed.), Mathematical Methods in Computer Graphics and Design. Academic Press, New York, pp. 63-86.

Watson, D.F., 1982. ACORD: Automatic contouring of raw data. Computers \& Geosciences 8 (1), 97-101.

Watson, D.F., 1992. Contouring: A Guide to the Analysis and Display of Spatial Data. Pergamon, Oxford, $321 \mathrm{pp.}$

Yates, S.R., 1987. CONTUR: a FORTRAN algorithm for two-dimensional high-quality contouring. Computers \& Geosciences 13 (1), 61-76. 